\title{
Glutathione s-transferase genotype protects against in utero tobacco linked lung function deficits
}

Louisa Owens ${ }^{1,2}$, Ingrid A Laing ${ }^{3,4}$, Jasminka Murdzoska ${ }^{3}$, Guicheng Zhang ${ }^{5,6}$, Steve W Turner ${ }^{7}$, Peter N Le Souëf ${ }^{1}$

1 School of Medicine University of Western Australia, GPO Box D184, Perth, Australia 2 School of Women's and Children's Health, University of New South Wales 2031, Australia 3 Telethon Kids Institute, 100 Roberts Road, Subiaco, Western Australia 6008, Australia 4 School of Biomedical Sciences, University of Western Australia, GPO Box D184, Perth, Australia 5 School of Public Health, Curtin University, Bentley, Western Australia 6845, Australia ${ }_{6}$ Centre for Genetic Origins of Health and Disease, University of Western Australia and Curtin University

7 School of Medical Sciences, University of Aberdeen, AB24 3FX, Scotland

Corresponding author:

Louisa Owens

Louisa.owens@health.nsw.gov.au

+61293821477

\section{Abbreviations}

FEF $25-75 \%$ - forced expiratory flow at $25-75 \%$ of forced vital capacity $\mathrm{FEV}_{1}$-forced expiratory volume in 1 second

FVC - forced vital capacity

GST - glutathione s-transferase

ITS - in utero tobacco smoke exposure

$\mathrm{PCR}$ - polymerase chain reaction

PIAF - Perth infant asthma follow-up study

ITS - in utero tobacco smoke

V'maxFRC - maximum flow at functional residual capacity 


\section{Abstract \\ Rationale}

In utero tobacco exposure is associated with reduced infant lung function. Anti-oxidant enzymes from the glutathione-s-transferase (GST) family may protect against these lung function deficits.

\section{Objectives:}

(1) Assess the long-term effect of in utero smoke exposure on lung function into adulthood.

(2) Assess whether GSTT1 and GSTMI active genotypes have longterm protective effects on lung function.

\section{Methods:}

In this longitudinal study, based on a normal population $(n=253)$, lung function was measured during infancy and then at 6,11,18 and 24 years. GSTM1 and GSTT1 genotype was analysed in a subgroup ( $n=179)$. Lung function was assessed longitudinally from 6 to 24 years $(n=144)$.

\section{Main Results}

Exposure to maternal in utero tobacco was associated with lower $\mathrm{FEV}_{1}$ and FVC from 6 to 24 years (mean difference $-3.87 \%$ predicted, $\mathrm{p}=0.021 ;-3.35 \%$ predicted, $\mathrm{p}=0.035$, respectively). Among those homozygous for the GSTM1 null genotype, in utero tobacco exposure was associated with lower $\mathrm{FEV}_{1}$ and FVC compared with those with no in utero tobacco exposure (mean difference $-6.2 \%$ predicted, $\mathrm{p}=0.01 ;-4.7 \%$ predicted, $\mathrm{p}=0.043$ respectively). For those with GSTM1 active genotype, there was no difference in lung function whether exposed to maternal in utero tobacco or not. In utero tobacco exposure was associated with deficits in lung function among those with both GSTT1 null and GSTT1 active genotypes. 


\section{Conclusions}

GST genotypes may have protective effects against the deficits in lung function associated with in utero tobacco exposure. This offers potential preventative targets in anti-oxidant pathways for at-risk infants of smoking mothers.

Abstract Words: 247 


\section{Introduction:}

The exposure of children's lungs to tobacco smoke is of grave concern as lungs are particularly vulnerable to insult during development. This exposure can occur while still in utero, as nicotine and other toxic substances freely pass through the placenta or postnatally in the home environment ${ }^{1}$.

Exposure to tobacco smoke antenatally is associated with reduced lung function during infancy and childhood ${ }^{34}$. Both ante- and post-natal smoke exposure are linked to an increase in respiratory symptoms throughout childhood and adolescence ${ }^{2}$.

We have previously shown that airway function measurements track from infancy into early adulthood indicating an inherent airway structure is laid down early in lung development, while measurements of adult lung size were independent of infant lung function, suggesting lung growth is modifiable by external factors.

Gene-environment interactions modulate the effects of in utero smoke (ITS) exposure on respiratory outcomes in childhood and fetal anti-oxidants may play a role in protecting the developing lungs. Activity levels of glutathione s-transferases (GST), a family of enzymes involved in the detoxification of xenobiotics, vary based on genotype, with homozygous deletions of the GSTT1 and GSTM1 genes associated with absent function of that particular enzyme $^{56}$. Among those exposed to ITS, GST active genotypes are linked to higher infant lung function when compared with those with the null genotype, as previously reported in this cohort

7. GST null genotypes are associated with increased risk of childhood asthma compared with those with active genotypes, particularly in the context of passive smoke exposure, suggesting an increased vulnerability to the detrimental effects of tobacco smoke ${ }^{8-10}$.

We hypothesised that the negative effect of ITS exposure on lung function would persist into adulthood but that this effect would be lessened for those with higher levels of innate 
detoxification enzymes. Our aim was to: (1) assess the long-term effects of both ante and postnatal smoke exposure on lung function, as a measure of lung development; and (2) assess whether GSTT1 and GSTM1 active genotypes are protective against the effects of in utero tobacco smoke on lung function through into adulthood.

\section{Methods and Materials:}

The Perth Infant Asthma Follow study was established in 1987. Over 24 months, 253 subjects were recruited antenatally from an urban maternity hospital in Perth, Western Australia. There was no preselection based on family history of asthma or atopy. Recruitment details have been published previously ${ }^{11}$. Infants were excluded if they were born premature <37 weeks gestation, had any major congenital abnormality or had any significant respiratory illnesses in the first month of life.

Detailed antenatal smoking history during each trimester of the pregnancy was collected from both parents. Fetal in utero tobacco exposure was classified as positive for maternal or paternal exposure if that parent smoked at all during the pregnancy. Subjects could be positive for both maternal and paternal in utero tobacco exposure. Subjects were classified as negative for any ITS exposure if neither parent smoked at all during the pregnancy.

Urinary cotinine, a byproduct of nicotine metabolism, was measured in a subgroup of infants at birth $(\mathrm{n}=85)$.

Subjects or their parents also completed a questionnaire at each follow-up assessment ${ }^{12}$. This included questions on history of physician-diagnosed asthma and tobacco smoke exposure in the household. "Postnatal smoke exposure only" was classified as a positive response to post- 
natal smoke exposure at either 1, 6 or 11 years of age and no history of maternal in utero smoke exposure. "Incomplete postnatal data" was recorded if there was less than 2 postnatal assessments.

The participants performed lung function testing at regular intervals from infancy through to young adulthood at 1 month, 6 months, 12 months, $6,11,18$ and 24 years of age.

The rapid thoraco-abdominal compression technique during tidal breathing of sedated infants was used during infant lung function testing ${ }^{13}$. The interaction between in utero tobacco exposure and GST genotype on infant lung function in this cohort has been published previously $^{7}$.

Spirometry was performed at each assessment from 6 to 24 years ${ }^{14}$. FEV 1 , FVC, FEF25-75\% and $\mathrm{FEV}_{1} / \mathrm{FVC}$ were recorded and converted into percent predicted scores based on sex, age, height and ethnicity using GLI reference values ${ }^{15}$.

GST genotyping analysis was performed on blood samples taken at either the 6 or 11 year assessments. GSTT1 and GSTM1 deletion polymorphisms were identified by polymerase chain reaction (PCR) methods as previously described in detail ${ }^{716}$. A subset of specimens had genotype results confirmed by PCR with a second set of primers ${ }^{7}$. CYP1Al primers were used as a positive control for each PCR performed. Those who were homozygous for the GST null genes were classified as GST null and those who were either heterozygous or homozygous for non-null GST genotype was classified as GST active.

The study was approved by the Western Australian Child and Adolescent Health Service Human Research Ethics Committee (2054EP). Parents, or subjects when appropriate (aged > 18 years), signed informed consent forms for each assessment. 


\section{Statistical analysis:}

Comparisons between the participants seen at each assessment was analysed by Pearson's chisquare for categorical variables and independent t-test for continuous variables.

Mean difference in cotinine levels between infants whose mothers reported smoking at recruitment and infants whose mothers reported not smoking at recruitment were measured using the Mann Whitney U test.

In the longitudinal analysis, the link between tobacco smoke exposure or GST genotype and lung function from 6 to 24 years were assessed by generalised estimating equations. These equations adjust for inherent covariance in each subject ${ }^{17}$.

Mean lung function results $\left(\mathrm{FEV}_{1}, \mathrm{FVC}, \mathrm{FEF} 25-75 \%\right.$ and $\mathrm{FEV}_{1} / \mathrm{FVC} \%$ predicted) from the 6 , 11, 18 and 24 year assessments were the longitudinal outcome variables for each participant. Maternal ITS, paternal ITS and postnatal tobacco exposure only were all assessed separately and compared with no in utero smoke exposure.

In order to assess the effect of GST polymorphisms on lung function in the context of in utero tobacco exposure, we then split the group into GST genotype i.e firstly GSTT1 null versus GSTT1 active and then GSTM1 null and GSTM1 active and applied the generalised estimating equations. Mean longitudinal lung function ( $\%$ predicted) for those exposed and not exposed to maternal ITS in each subgroup were included as outcome variables.

Two-sided $p$ value $<0.05$ determined statistical significance.

This study had a power of 0.813 to reject the null hypothesis of no significant difference in $\mathrm{FEV}_{1}$ between GSTT1 null and GSTT1 non-null genotype groups based on 411 assessments, if the real difference between groups was 5\% predicted, with standard deviation of $12 \%$ predicted. The Type I error probability associated with this test of this null hypothesis is 0.05 . 
Analyses were performed using SPSS Statistics for Windows, version 24.0. (2016, Armonk, NY: IBM Corp).

\section{Results:}

\section{General}

Of the original 253 subjects recruited, smoking history during the pregnancy was collected from 252 mothers and 240 fathers. Eighty five subjects (34\%) reported exposure to maternal smoking during the pregnancy and ninety-four (39\%) reported exposure to paternal smoking during the pregnancy. A further 47 subjects out of 129 with no maternal ITS exposure and at least 2 postnatal assessments, reported exposure to post-natal tobacco smoke in the home, with 82 subjects reporting no pre or post natal smoke exposure and incomplete post-natal exposure data on the remaining 38 subjects.

Lung function testing was performed on 110 subjects at 6 years, 183 at 11 years, 141 at 18 years and 118 subjects at 24 years. Comparison of subject characteristics at each assessment have previously been published ${ }^{18}$. The only significant difference between the original cohort and those seen at follow up was less parental ITS exposure in those seen at later follow ups (54\% ITS exposure in original cohort; $45 \%$ of those seen at 18 years; $43 \%$ of those seen at 24 years). GSTT1 and GSTM1 genotyping was performed on 179 subjects and the frequency of each genotype is presented in table 1 . There were 144 subjects with 443 assessments included in the longitudinal analysis of smoke exposure, and 128 subjects with 411 assessments in the longitudinal analysis of GST genotype and smoke exposure.

$\underline{\text { Cotinine levels and maternal smoke exposure }}$ 
Neonatal cotinine levels were higher amongst infants whose mothers reported smoking at recruitment during pregnancy $(\mathrm{n}=25$; mean $75.3 \mathrm{ng} / \mathrm{ml}$ creatinine, SD 57.7), than those whose mothers reported no smoking at recruitment ( $\mathrm{n}=60$; mean $9.3 \mathrm{ng} / \mathrm{ml}$ creatinine, SD 30.5), $\mathrm{p}<0.001$.

\section{$\underline{\text { In utero tobacco smoke exposure and lung function }}$}

Exposure to maternal ITS was associated with significantly lower FEV 1 and FVC from 6 to 24 years of age (mean difference $-3.87 \%$ predicted, $\mathrm{p}=0.021$, and $-3.35 \%$ predicted, $\mathrm{p}=0.035$, respectively) Figure 1; supplementary table 1. Exposure to maternal ITS was not associated with a difference in either $\mathrm{FEV}_{1} / \mathrm{FVC}$ or FEF25-75\%. Neither exposure to paternal ITS nor postnatal tobacco exposure only were associated with significant changes in lung function from 6 to 24 years. Therefore, further reference to ITS refers to maternal ITS exposure only, independent of post-natal smoke exposure.

\section{$\underline{G S T \text { polymorphisms and in utero tobacco smoke exposure }}$}

There was no difference in lung function from 6 to 24 years of age between the GST null and active genotype groups (supplementary table 2).

Among those with the GSTM1 active genotype, maternal ITS exposure was not associated with any significant difference in lung function from 6 and 24 years of age. Among those with the GSTM1 null genotype, ITS exposure was associated with a lower $\mathrm{FEV}_{1}$ and FVC compared with those with no ITS exposure (mean difference $\mathrm{FEV}_{1}-6.2 \%$ predicted, $\mathrm{p}=0.01$; mean difference FVC $-4.7 \%$ predicted, $\mathrm{p}=0.043$ ) (table 2; figure 2) 
Among those with the GSTT1 active genotype, $\mathrm{FEV}_{1}$ and $\mathrm{FVC}$ were lower in those exposed to maternal ITS compared with no maternal ITS exposure (mean difference $\mathrm{FEV}_{1}=-4.05 \%$ predicted, $\mathrm{p}=0.034 ; \mathrm{FVC}=-3.7 \%$ predicted, $\mathrm{p}=0.037$ respectively). Among those with the GSTT1 null genotype, $\mathrm{FEV}_{1}$ and FEF25-75\% were lower in those exposed to ITS, compared with no ITS (mean difference $\mathrm{FEV}_{1}=-10.29 \%$ predicted, $\mathrm{p}=0.021$; mean difference FEF25$75 \%=-15.2 \%$ predicted, $\mathrm{p}=0.008$ ). (table 2 ; figure 2 ). However, only three subjects with exposure to maternal ITS had the GSTT1 null genotype and all three also had the GSTM1 null genotype.

\section{Discussion:}

This longitudinal, birth-cohort study of lung function confirms that maternal ITS exposure is linked to lower lung function from infancy to early adulthood, and establishes an important new finding: the major effect of in utero smoke exposure is on lung size rather than airway size or function. This conclusion stems from the finding that the deficits in future respiratory function were specifically in $\mathrm{FEV}_{1}$ and $\mathrm{FVC}$ and not $\mathrm{FEF} 25-75 \%$ or $\mathrm{FEV}_{1} / \mathrm{FVC}$. A previous study from this cohort revealed that variables reflecting airway function, V'maxFRC in infancy and $\mathrm{FEF} 25-75 \%$ and $\mathrm{FEV}_{1} / \mathrm{FVC}$ thereafter, track from infancy into early adulthood. Together these findings provide compelling evidence suggesting that the foundations of airway structure are laid down during antenatal development, persist throughout childhood and are relatively resistant to environmental insults. In contrast, lung size in childhood, as measured by $\mathrm{FEV}_{1}$ and FVC, had no correlation with infant airway function, but did correlate with tobacco smoke exposure, implying that lung size (perhaps as a reflection of alveolar number) is vulnerable to external, environmental factors. 
A further important finding was evidence suggesting that the presence of active glutathione stransferase enzymes provide longterm protection from the damage caused by maternal ITS exposure. In particular, the functional GSTM1 active genotype appears to have this protective effect. Maternal ITS was only associated with deficits in lung function up to adulthood for those with the GSTM1 null genotype and not for those with the GSTM1 active genotype. The functional GSTT1 active genotype did not appear to share this degree of protective effect, as those with this genotype still had significant deficits in lung function if exposed to maternal ITS compared with those with no ITS exposure. However, the difference between those exposed and not exposed was smaller than among the GSTT1 null genotype group suggesting that GSTT1 active may still provide a degree of protection, although not enough to fully overcome the damage associated with ITS exposure. GSTT1 may be of more importance in protection very early on, as in a previous study from this cohort, GSTT1 active genotype was associated with higher lung function during infancy mong those exposed to maternal ITS than the GSTT1 null group ${ }^{7}$.

Glutathione s-transferase is an enzyme which catalyses the reaction between glutathione and electrophilic xenobiotics and reactive oxygen species, making it crucial to the body's detoxification processes ${ }^{19}$. There are eight classes of cytosolic GST in humans, each with several subclasses, which vary in their structure and substrate specificity ${ }^{20}$. Although nicotine is not metabolised by the GST enzymes, many of the other toxic substances within cigarettes $\operatorname{are}^{2122}$. Homozygous GSTM1 and GSTT1 null polymorphisms have been associated with the development of asthma in childhood, thought to be due to an increase in oxidative stress, although they are not related to asthma severity (turner new paper). Adjusting for confounding variables during childhood such as tobacco exposure and environmental pollution is difficult and may be the reason results from previous studies have not been convincing ${ }^{23}$. GSTM1 and GSTT1 null genotypes have both been found to be strong predictors of COPD in adult 
females $^{24}$. The GSTM1 null genotype is also associated with reduced lung function growth in children, while the GSTT1 null genotype is associated with accelerated lung function decline in adult males ${ }^{25} 26$. However, these studies did not specifically investigate those exposed to tobacco smoke in utero, a critical time during lung development and we did not find a link between GST genotype and lung function among those not exposed to ITS.

The GSTT1 null genotype is rarer than the GSTM1 null genotype, affecting only $15 \%$ of the population. The subject numbers with both GSM1 null genotype and maternal ITS exposure, $\mathrm{n}=3$, make definitive statements about this group difficult. However, the length of follow up from infancy through into early adulthood in this study is an important advantage, as this spans the entire post-natal lung growth phase, to the peak in lung function in early adulthood.

During the in utero period of organogenesis, the fetus is exposed to the same levels of nicotine and other toxic substances as found in the actively-smoking mother, as these toxic substances pass freely through the placenta. Cotinine, a by-product of nicotine metabolism, can be measured in umbilical cord blood of newborn infants whose mothers smoked during pregnancy ${ }^{1}$. Although the tobacco is not inhaled into the lungs of the fetus, the serum exposure to these toxins is associated with reduced lung function when measured in the first few days of life, even before any post-natal tobacco smoke expsoure ${ }^{27}$. Given the negative associations between tobacco exposure while pregnant and infant outcomes, pregnant mothers may be reluctant to admit smoking to study researchers. However we collected objective evidence of tobacco exposure, with neonatal urinary cotinine levels, which confirm the reliability of the parent reported smoking data.

Interestingly, there was no link between either paternal ITS exposure or postnatal tobacco smoke exposure alone, on lung function throughout childhood, suggesting the most 
significant impact of tobacco exposure on lung function is a dose response effect during the in utero developmental phase.

Children with two hits, a toxic exposure and a genetic vulnerability, are at risk for the largest deficits in lung growth during lung development. Our data suggests there is a protective benefit in having higher anti-oxidant levels during in utero development and this warrants further exploration. Finding a way to protect the lungs during critical periods of development, for example with anti-oxidant supplementation or boosting the fetal glutathione pathway, and avoiding long-term detrimental consequences could potentially be an important target in minimising chronic respiratory morbidity for the children of smoking mothers.

\section{Acknowledgements:}

We would like to thank all the previous contributors to the Perth Infant Asthma Follow up study including David Mullane, Desmond Cox, Kimberley Franks, Lou Landau, Jack Goldblatt, Sally Young, Siew-Kim Khoo, Neil Gibson, Veena Judge, Lyle Palmer, Paul O'Keefe, Jackie Arnott, Steve Stick, Peter Rye, Catherine Hayden and Sunalene Devadason.

\section{References:}

1. Wu FY, Chiu HT, Wu HD, et al. Comparison of urinary and plasma cotinine levels during the three trimesters of pregnancy. Paediatric and perinatal epidemiology 2008;22(3):296-301.

2. Burke H, Leonardi-Bee J, Hashim A, et al. Prenatal and passive smoke exposure and incidence of asthma and wheeze: systematic review and meta-analysis. Pediatrics 2012;129(4):735-44. 
3. Turner S, Fielding S, Mullane D, et al. A longitudinal study of lung function from 1 month to 18 years of age. Thorax 2014;69(11):1015-20.

4. Li YF, Gilliland FD, Berhane K, et al. Effects of in utero and environmental tobacco smoke exposure on lung function in boys and girls with and without asthma. American journal of respiratory and critical care medicine 2000;162(6):2097-104.

5. Board P, Coggan M, Johnston P, et al. Genetic heterogeneity of the human glutathione transferases: a complex of gene families. Pharmacology \& therapeutics 1990;48(3):357-69.

6. Pemble S, Schroeder KR, Spencer SR, et al. Human glutathione S-transferase theta (GSTT1): cDNA cloning and the characterization of a genetic polymorphism. The Biochemical journal 1994;300 ( Pt 1):271-6.

7. Murdzoska J, Devadason SG, Khoo S-K, et al. In Utero Smoke Exposure and Role of Maternal and Infant Glutathione S-Transferase Genes on Airway Responsiveness and Lung Function in Infancy. American journal of respiratory and critical care medicine 2010;181(1):64-71.

8. Ivaschenko TE, Sideleva OG, Baranov VS. Glutathione- S-transferase micro and theta gene polymorphisms as new risk factors of atopic bronchial asthma. Journal of molecular medicine (Berlin, Germany) 2002;80(1):39-43.

9. Gilliland FD, Li YF, Peters JM. Effects of maternal smoking during pregnancy and environmental tobacco smoke on asthma and wheezing in children. American journal of respiratory and critical care medicine 2001;163(2):429-36.

10. Kabesch M, Hoefler C, Carr D, et al. Glutathione S transferase deficiency and passive smoking increase childhood asthma. Thorax 2004;59(7):569-73. 
11. Young S, Le Souef PN, Geelhoed GC, et al. The influence of a family history of asthma and parental smoking on airway responsiveness in early infancy. The New England journal of medicine 1991;324(17):1168-73.

12. Ferris BG. Epidemiology Standardization Project (American Thoracic Society). Am Rev Respir Dis 1978;118(6 Pt 2):1-120.

13. Sly PD, Tepper R, Henschen M, et al. Tidal forced expirations. ERS/ATS Task Force on Standards for Infant Respiratory Function Testing. European Respiratory Society/American Thoracic Society. The European respiratory journal 2000;16(4):741-8.

14. Miller MR, Hankinson J, Brusasco V, et al. Standardisation of spirometry. The European respiratory journal 2005;26(2):319-38.

15. Quanjer PH, Stanojevic S, Cole TJ, et al. Multi-ethnic reference values for spirometry for the 3-95-yr age range: the global lung function 2012 equations. The European respiratory journal 2012;40(6):1324-43.

16. Harries LW, Stubbins MJ, Forman D, et al. Identification of genetic polymorphisms at the glutathione S-transferase Pi locus and association with susceptibility to bladder, testicular and prostate cancer. Carcinogenesis 1997;18(4):641-44.

17. K-Y Liang Zeger SL. Longitudinal data analysis using generalized linear models. Biometrika 1986; 73: 13-22.

18. Owens L, Laing I, Zhang G, et al. Early sensitization is associated with reduced lung function from birth into adulthood. The Journal of allergy and clinical immunology 2016;137(5):1605-07.e2.

19. Li X. Glutathione and Glutathione-S-Transferase in Detoxification Mechanisms. General, Applied and Systems Toxicology: John Wiley \& Sons, Ltd 2009. 
20. Sheehan D, Meade G, Foley VM, et al. Structure, function and evolution of glutathione transferases: implications for classification of non-mammalian members of an ancient enzyme superfamily. The Biochemical journal 2001;360(Pt 1):1-16.

21. Hukkanen J, Jacob P, Benowitz NL. Metabolism and Disposition Kinetics of Nicotine. Pharmacological Reviews 2005;57(1):79.

22. Berhane K, Widersten M, Engström A, et al. Detoxication of base propenals and other alpha, beta-unsaturated aldehyde products of radical reactions and lipid peroxidation by human glutathione transferases. Proceedings of the National Academy of Sciences 1994;91(4):1480-84

23. Minelli C, Granell R, Newson R, et al. Glutathione-S-transferase genes and asthma phenotypes: a Human Genome Epidemiology (HuGE) systematic review and metaanalysis including unpublished data. International journal of epidemiology 2010;39(2):539-62.

24. Malic Z, Topic A, Francuski D, et al. Oxidative Stress and Genetic Variants of Xenobiotic-Metabolising Enzymes Associated with COPD Development and Severity in Serbian Adults. Copd 2017;14(1):95-104.

25. Imboden M, Downs SH, Senn O, et al. Glutathione S-transferase genotypes modify lung function decline in the general population: SAPALDIA cohort study. Respiratory Research 2007;8(1):2.

26. Gilliland FD, Gauderman WJ, Vora H, et al. Effects of glutathione-S-transferase M1, T1, and P1 on childhood lung function growth. American journal of respiratory and critical care medicine 2002;166

27. Lodrup Carlsen KC, Jaakkola JJ, Nafstad P, et al. In utero exposure to cigarette smoking influences lung function at birth. The European respiratory journal 1997;10(8):17749. 
Table 1. Prevalence of GST genotypes $(n=179)$

\begin{tabular}{ll}
\hline & $\mathbf{N}$ \\
\hline GSTM1 - & $105(59 \%)$ \\
GSTM1 + & $74(41 \%)$ \\
GSTT1 - & $27(15 \%)$ \\
GSTT1 + & $152(85 \%)$ \\
GSTM1 - and GSTT1 - & $11(6 \%)$
\end{tabular}

$-=$ homozygous null genotype; $+=$ heterozygous or homozygous non-null genotype.

Figure 1. Mean lung function from 6-24 years by tobacco smoke exposure. Number of subjects=144; number of assessments $=443$

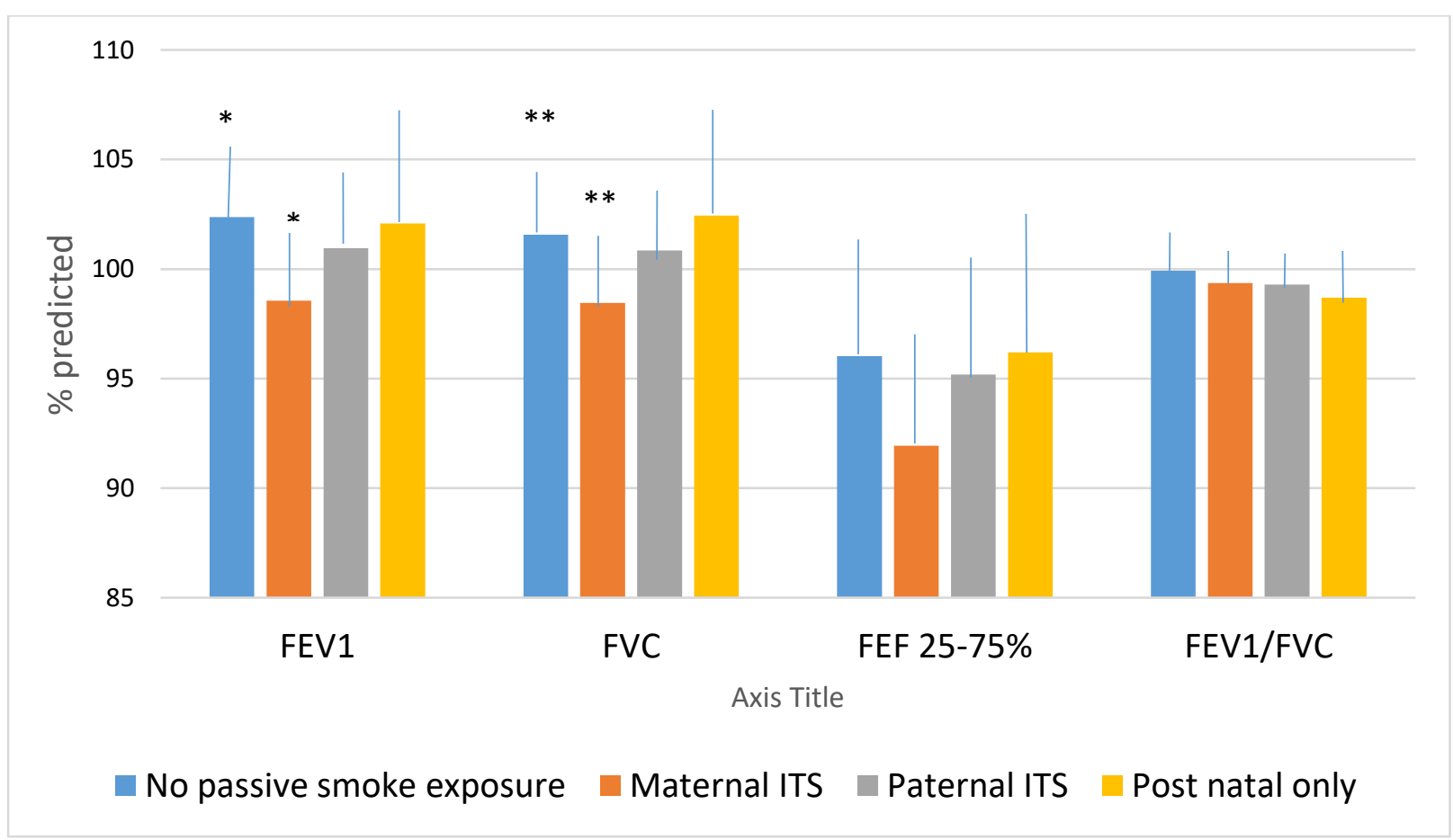

Lung function from 6-24 years. \% predicted based on GLI reference values. Generalised estimating equations. Error bars depicting $95 \%$ confidence interval.

$* \mathrm{p}=0.021 ; * * \mathrm{p}=0.035$ 
Table 2. Association between maternal in utero tobacco smoke exposure and lung function from 6-24 years, grouped by GST genotype.

\begin{tabular}{|c|c|c|c|c|c|}
\hline & & $\begin{array}{l}\text { FEV }_{1} \% \text { pred } \\
(95 \% \text { CI })\end{array}$ & $\begin{array}{l}\text { FVC \% pred } \\
(95 \% \text { CI })\end{array}$ & $\begin{array}{c}\text { FEF 25-75\% } \\
\text { pred }\end{array}$ & $\begin{array}{c}\text { FEV }_{1} / \mathbf{F V C} \% \\
\text { pred } \\
(95 \% \mathrm{CI})\end{array}$ \\
\hline \multirow[t]{2}{*}{ GSTM1 null } & $\begin{array}{l}\text { No ITS } \\
(\mathrm{n}=52)\end{array}$ & $\begin{array}{c}102.88 \\
(100.02-105.73)\end{array}$ & $\begin{array}{c}101.77 \\
(99.04-104.5)\end{array}$ & $\begin{array}{c}97.88 \\
(93-102.75)\end{array}$ & $\begin{array}{c}100.22 \\
(98.8-101.6)\end{array}$ \\
\hline & $\begin{array}{l}\text { Maternal ITS } \\
(\mathrm{n}=23)\end{array}$ & $\begin{array}{c}96.7 \\
(92.92-100.49) \\
p=0.01\end{array}$ & $\begin{array}{c}97.06 \\
(93.4-100.72) \\
p=0.043\end{array}$ & $\begin{array}{c}90.36 \\
(83.85-96.89) \\
p=0.07\end{array}$ & $\begin{array}{c}98.88 \\
(97.0-100.8) \\
p=0.27\end{array}$ \\
\hline \multirow[t]{2}{*}{$\begin{array}{l}\text { GSTM1 non- } \\
\text { null }\end{array}$} & $\begin{array}{l}\text { No ITS } \\
(\mathrm{n}=41)\end{array}$ & $\begin{array}{c}102.56 \\
(99.51-105.61)\end{array}$ & $\begin{array}{c}102.83 \\
(99.82-105.84)\end{array}$ & $\begin{array}{c}95.38 \\
(90.06-100.69)\end{array}$ & $\begin{array}{c}98.78 \\
(96.87-100.69)\end{array}$ \\
\hline & $\begin{array}{l}\text { Maternal ITS } \\
(\mathrm{n}=12)\end{array}$ & $\begin{array}{c}100.5 \\
(96.63-104.37) \\
\mathrm{p}=0.41\end{array}$ & $\begin{array}{c}100.05 \\
(96.68-103.43) \\
p=0.229\end{array}$ & $\begin{array}{c}92.54 \\
(85.18-99.89) \\
p=0.54\end{array}$ & $\begin{array}{c}99.78 \\
(97.48-102.1) \\
\mathrm{p}=0.51\end{array}$ \\
\hline \multirow[t]{2}{*}{ GSTT1 null } & No ITS $(n=18)$ & $\begin{array}{c}102.26 \\
(98.64-105.88)\end{array}$ & $\begin{array}{c}100.44 \\
(95.96-104.92)\end{array}$ & $\begin{array}{c}100.84 \\
(94.73-106.96)\end{array}$ & $\begin{array}{c}100.74 \\
(98.19-103.29)\end{array}$ \\
\hline & $\begin{array}{l}\text { Maternal ITS } \\
(\mathrm{n}=3)\end{array}$ & $\begin{array}{c}91.94 \\
(83.98-99.9) \\
p=0.021\end{array}$ & $\begin{array}{c}91.74 \\
(80.78-102.7) \\
p=0.15\end{array}$ & $\begin{array}{c}85.63 \\
(76.2-95.08) \\
p=0.008\end{array}$ & $\begin{array}{c}99.8 \\
(95.51-104.09) \\
p=0.71\end{array}$ \\
\hline \multirow[t]{2}{*}{$\begin{array}{l}\text { GSTT1 non- } \\
\text { null }\end{array}$} & $\begin{array}{l}\text { No ITS } \\
(\mathrm{n}=75)\end{array}$ & $\begin{array}{c}102.85 \\
(100.43-105.26)\end{array}$ & $\begin{array}{c}102.6 \\
(100.34-104.86)\end{array}$ & $\begin{array}{c}95.96 \\
(91.8-100.12)\end{array}$ & $\begin{array}{c}99.37 \\
(98.08-100.65)\end{array}$ \\
\hline & $\begin{array}{l}\text { Maternal ITS } \\
(\mathrm{n}=32)\end{array}$ & $\begin{array}{c}98.8 \\
\left(\begin{array}{c}95.94-101.67) \\
p=0.034\end{array}\right.\end{array}$ & $\begin{array}{c}98.9 \\
(96.32-101.4) \\
p=0.031\end{array}$ & $\begin{array}{c}91.75 \\
(86.49-97.01) \\
p=0.22\end{array}$ & $\begin{array}{c}99.19 \\
(97.63-100.75) \\
p=0.86\end{array}$ \\
\hline
\end{tabular}

Mean difference in \% predicted lung function for those exposed to maternal in utero tobacco smoke, compared with not exposed to maternal in utero tobacco smoke, by GST genotype. Number of subjects $=128$; number of assessments $=411$ Bold font indicates $p<0.05$ 
Figure 2. Mean lung function from 6-24 years for GST null versus non-null genotype, by maternal ITS exposure
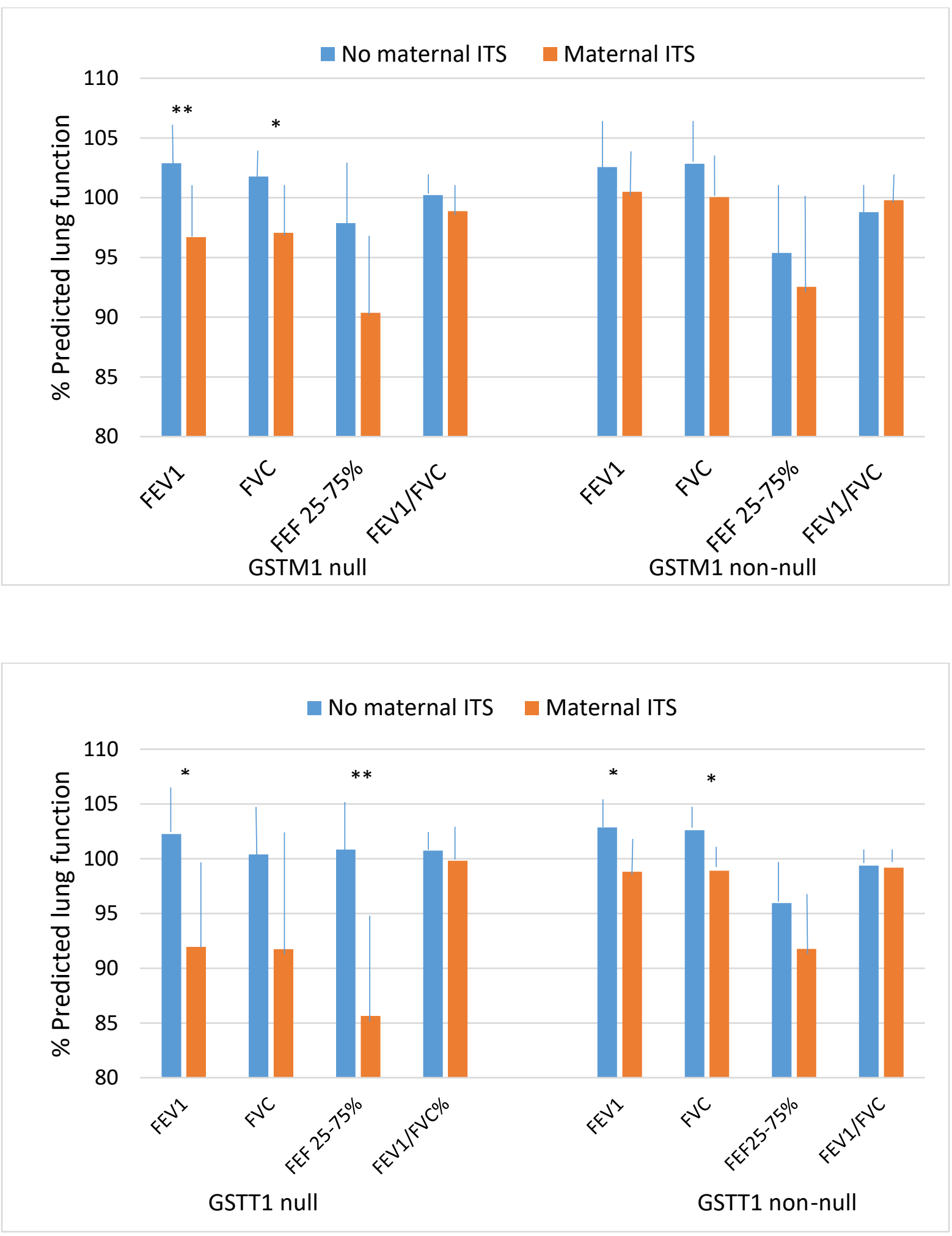

Mean lung function longitudinally from 6-24 years, \% predicted based on GLI reference range. ${ }^{*} \mathrm{p}<0.05 ;{ }^{* *} \mathrm{p} \leq 0.01$ 
Supplementary Table 1: Lung function from 6-24 years by passive smoke exposure

\begin{tabular}{lcccc}
\hline & $\begin{array}{c}\text { FEV1\% } \\
\text { predicted }\end{array}$ & $\begin{array}{c}\text { FVC } \\
\text { \% predicted }\end{array}$ & $\begin{array}{c}\text { FEF25-75\% } \\
\text { \% predicted }\end{array}$ & $\begin{array}{c}\text { FEV1/FVC } \\
\text { \% predicted }\end{array}$ \\
\hline $\begin{array}{l}\text { No passive } \\
\text { smoke exposure }\end{array}$ & $\begin{array}{l}102.37 \\
(99.83-104.91)\end{array}$ & $\begin{array}{c}101.57 \\
(99.16-103.97)\end{array}$ & $\begin{array}{c}96.02 \\
(91.67-100.36)\end{array}$ & $\begin{array}{c}99.93 \\
(98.56-101.31)\end{array}$ \\
Maternal ITS & 98.55 & 98.45 & 91.94 & 99.36 \\
& $(95.88-101.22)$ & $(95.97-100.93)$ & $(87.21-96.67)$ & $(97.95-100.77)$ \\
Paternal ITS & 100.95 & 100.84 & 95.18 & 99.29 \\
& $(98.34-103.56)$ & $(98.29-103.39)$ & $(90.89-99.47)$ & $(98.01-100.57)$ \\
Postnatal smoke & 102.08 & 102.44 & & 98.69 \\
exposure only & $(97.87-106.29)$ & $(98.23-106.65)$ & $(89.46-102.9)$ & $(96.5-100.87)$ \\
\hline
\end{tabular}

No passive smoke exposure - no in utero or postnatal tobacco smoke exposure from either parent or household member; Maternal ITS - mother smoked at all during the pregnancy; Paternal ITS - father smoked at all during the pregnancy; Postnatal smoke exposure only- no maternal in utero tobacco smoke exposure, but postnatal smoke exposure in the home.

Supplementary Table 2: GST polymorphisms and lung function from 6 to 24 years

\begin{tabular}{lcccc}
\hline & $\begin{array}{c}\text { Mean FEV } \\
\text { predicted }\end{array}$ & $\begin{array}{c}\text { Mean FVC\% } \\
\text { predicted }\end{array}$ & $\begin{array}{c}\text { Mean FEF25- } \\
\mathbf{7 5 \%} \\
\text { predicted }\end{array}$ & $\begin{array}{c}\text { Mean } \\
\text { FEV1/FVC } \\
\text { \% predicted }\end{array}$ \\
\hline GSTT1 null & 100.45 & 98.91 & 98.17 & 100.57 \\
GSTT1 non- & 101.52 & 101.37 & $(92.47-103.87)$ & $(98.34-102.8)$ \\
null & $(99.61-103.43)$ & $(99.61-103.14)$ & $(91.28-97.9)$ & $(98.3-100.31)$ \\
& $\mathrm{p}=0.62$ & $\mathrm{p}=0.32$ & $\mathrm{p}=0.29$ & $\mathrm{p}=0.31$ \\
GSTM1 null & 100.94 & 100.29 & & \\
& $(98.56-103.3)$ & $(98.04-102.54)$ & $(91.55-99.51)$ & $(98.65-100.94)$ \\
GSTM1 non- & 101.96 & 102.02 & 94.56 & 99.79 \\
null & $(99.5-104.42)$ & $(99.64-104.4)$ & $(90.2-98.9)$ & $(97.56-100.59)$ \\
& & & & $\mathrm{p}=0.46$ \\
& $\mathrm{p}=0.56$ & $\mathrm{p}=0.3$ & $\mathrm{p}=0.75$ & \\
\hline
\end{tabular}

Mean lung function from 6-24 years by GST genotype. \% predicted based on GLI reference values. Unadjusted values 
